\title{
Endoscopic evaluation of celiac disease
}

Authors

Institution
Giovanni Cammarota, Gianluca laniro

Internal Medicine, Gastroenterology and Liver Unit; “A. Gemelli” University Hospital, Rome, Italy submitted

19. February 2016 accepted after revision 14. March 2016

\section{Bibliography}

DOI http://dx.doi.org/

10.1055/s-0042-105435

Published online: 15.4.2016

Endoscopy International Open

2016; 04: E547-E548

(c) Georg Thieme Verlag KG

Stuttgart $\cdot$ New York

E-ISSN 2196-9736

\section{Corresponding author}

\section{Prof. Giovanni Cammarota,}

MD

Gemelli University Hospital Internal Medicine,

Gastroenterology and Liver Unit

Largo A. Gemelli

8, 00168 - Roma

Italia

Fax: +39-06-35502775

giovanni.cammarota@unicatt.it
Although serology-based diagnosis of celiac disease (CD) in children recently has been legitimized [1], small bowel biopsy remains the gold standard for diagnosis of the condition $[2,3]$. Upper endoscopy, therefore, takes on paramount importance in management of $C D$ for several reasons, including serendipitous discovery of endoscopic markers of $C D$, assessment of "patchy" villous atrophy, targeting of biopsy sampling, and evaluation of CD-related complications.

Macroscopic markers of $C D$, estimable with white light endoscopy, include the "scalloped" appearance of duodenal folds, nodular pattern of the mucosa (the so-called "mosaicism"), evidence of submucosal vessels, and epithelial fissurations [4]. The diagnostic accuracy of these findings is largely variable, according to different reports $[5,6]$, and they are associated with a significant rate of underdiagnosis of $\mathrm{CD}[7,8]$. Because standard endoscopy is unreliable, other endoscopic tools have been investigated for diagnosis opf $C D$, which fall into two categories based on their working principle: machine-independent techniques and machine-dependent techniques; the latter include software- and hardware-dependent techniques $[9,10]$. The water-immersion technique (WIT) and dye-staining chromoendoscopy and machine-independent. Software-dependent techniques include Narrow-Band Imaging (optical dye-less chromoendoscopy), Fujinon Intelligent Chromo Endoscopy (virtual dye-less chromoendoscopy) and i-SCAN, which are dyeless chromoendoscopy tools. Hardware-dependent techniques such as optical coherence tomography, confocal laser endomicroscopy, video capsule endoscopy, and enteroscopy can be performed only with dedicated tools that are different from regular gastroscopes, or with the use of probes $[9,10]$. The combination of these techniques has been suggested to improve the detection of duodenal villous abnormalities [11].
In this issue of Endoscopy International Open, Iacucci et al [12] present a retrospective cohort study of 58 patients with clinical suspicion of $C D$ and positive serology testings who underwent upper endoscopy and duodenal evaluation with both white light endoscopy (WLE) and a combination of i-SCAN and WIT (iSCAN-HDWI). The duodenal view was respectively classified as normal, reduction of folds, mosaic pattern, scalloping and atrophy with visible vessels with WLE, and as normal, mild, moderate, patchy or severe villous atrophy with iSCAN-HDWI.

The authors found a significant correlation between the endoscopic grade evaluated by iSCANHDWI and the histology score. Assessment with WLE showed a lower but significant grade of correlation. In particular, iSCAN-HDWI achieved 96\% sensitivity, $63 \%$ specificity, and $100 \%$ accuracy for predicting duodenal damage (excluding Marsh I lesions), whereas WLE showed 78\% sensitivity, $50 \%$ specificity, and $72 \%$ accuracy for the same gold standard. Respectively, WLE identified no abnormalities in $55.6 \%$ of patients diagnosed with patchy villous atrophy and in $33.3 \%$ of patients diagnosed with mild villous atrophy after iSCAN-HDWI evaluation.

WIT is an easy technique that allows real-time enhancement of duodenal villous pattern during upper endoscopy. After aspiration of air from the duodenal lumen, the operator injects $100 \mathrm{~mL}$ to $150 \mathrm{~mL}$ of water to highlight villi [13]. WIT achieved high levels of accuracy in diagnosing total villous atrophy (TVA), with only slightly less accurate results in identifying partial villous atrophy (PVA) [14-17].

i-SCAN is a digital tool developed by Pentax Medical. It enhances images through three different modalities: contrast enhancement, which highlights mucosal abnormalities, particularly those of depressed areas; surface enhancement, which increases contrast between light and dark; and tone enhancement, which groups and recom- 
bines blue, red, and green components of images. i-SCAN produced results similar to WIT in assessment of TVA and PVA [18].

The combination of iSCAN technology with WIT both highlights vascular and mucosal pattern and allows direct visualization of villi. Such a "joint-venture" can be of help in evaluating the duodenal villous pattern, especially in the case of partial or patchy villous atrophy, and in targeting biopsy sampling; therefore, it is advocated to decrease the number of $C D$ misdiagnoses and related unnecessary costs. Further studies, combining other modalities for imaging enhancement, are therefore welcome to improve our knowledge of the diagnostic potential of endoscopic tools in CD.

\section{Competing interests: None}

\section{References}

1 Husby S, Koletzko S, Korponay-Szabó IR. European Society for Pediatric Gastroenterology, Hepatology, and Nutrition guidelines for the diagnosis of coeliac disease. et al. ESPGHAN Working Group on Coeliac Disease Diagnosis; ESPGHAN Gastroenterology Committee; European Society for Pediatric Gastroenterology, Hepatology, and Nutrition. J Pediatr Gastroenterol Nutr 2012; 54: 136-160

2 Rubio-Tapia A, Hill ID, Kelly CP. American College of Gastroenterology. et al. ACG clinical guidelines: diagnosis and management of celiac disease. Am J Gastroenterol 2013; 108: 656-676

3 Ludvigsson JF, Bai JC, Biagi F. BSG Coeliac Disease Guidelines Development Group; British Society of Gastroenterology. et al. Diagnosis and management of adult coeliac disease: guidelines from the British Society of Gastroenterology. Gut 2014; 63: 1210-1228

4 Brocchi E, Tomassetti P, Misitano B et al. Endoscopic markers in adult coeliac disease. Dig Liver Dis 2002; 34: 177-182

5 Maurino E, Capizzano H, Niveloni S et al. Value of endoscopic markers in celiac disease. Digestive diseases and sciences 1993; 38: 2028-2033

6 Lecleire S, Di Fiore F, Antonietti $M$ et al. Endoscopic markers of villous atrophy are not useful for the detection of celiac disease in patients with dyspeptic symptoms. Endoscopy 2006; 38: 696-701
7 Barada K, Habib RH, Malli A et al. Prediction of celiac disease at endoscopy. Endoscopy 2014; 46: 110-119

8 Robson K, Alizart M, Martin J et al. Coeliac patients are undiagnosed at routine upper endoscopy. PloS one 2014; 9: e90552

9 Ianiro G, Gasbarrini A, Cammarota G. Endoscopic tools for the diagnosis and evaluation of celiac disease. World J Gastroenterol 2013; 19 : $8562-8570$

10 Cammarota G, Fedeli P, Gasbarrini A. Emerging technologies in upper gastrointestinal endoscopy and celiac disease. Nat Clin Pract Gastroenterol Hepatol 2009; 6: 47-56

11 Fedeli P, Gasbarrini G, Cammarota G. The combined application of advanced endoscopic imaging techniques may increase the duodenal villous morphology definition in suspected celiac disease. Dig Liver Dis 2010; 42: 595-596

12 Iacucci M, Poon T, Gui XS et al. High definition iSCAN endoscopy with water immersion technique accurately reflects histological severity of Celiac Disease. Endoscopy International Open 2016

13 Gasbarrini A, Ojetti V, Cuoco L et al. Lack of endoscopic visualization of intestinal villi with the "immersion technique" in overt atrophic celiac disease. Gastrointestinal Endoscopy 2003; 57: 348-351

14 Cammarota G, Pirozzi GA, Martino A et al. Reliability of the "immersion technique" during routine upper endoscopy for detection of abnormalities of duodenal villi in patients with dyspepsia. Gastrointestinal Endoscopy 2004; 60: 223-228

15 Cammarota G, Cuoco L, Cesaro $P$ et al. A highly accurate method for monitoring histological recovery in patients with celiac disease on a gluten-free diet using an endoscopic approach that avoids the need for biopsy: A double-center study. Endoscopy 2007; 39: 46-51

16 Cammarota G, Cesaro P, La Mura R et al. Role of the "immersion technique" in diagnosing celiac disease with villous atrophy limited to the duodenal bulb. Journal of Clinical Gastroenterology 2007; 41: 571 575

17 Cammarota G, Cazzato A, Genovese $O$ et al. Water-immersion technique during standard upper endoscopy may be useful to drive the biopsy sampling of duodenal mucosa in children with celiac disease. Journal of Pediatric Gastroenterology and Nutrition 2009; 49: 411 - 416

18 Cammarota G, Ianiro G, Sparano $L$ et al. Image-enhanced endoscopy with i-scan technology for the evaluation of duodenal villous patterns, Dig. Dis. Sci 2013; 58: 1287-1292 